\title{
El costo en salud y la corresponsabilidad clínica desde un enfoque gerencial
}

\author{
Health cost and clinical co-responsibility from a management \\ approach
}

\section{Orlando Carnota Lauzán}

Doctor en Ciencias Económicas. Investigador de Mérito. Profesor Titular. Escuela Nacional de Salud Pública. La Habana, Cuba.

\section{RESUMEN}

El trabajo enfoca el costo en salud, y particularmente su crecimiento, bajo una óptica gerencial, lo que implica destacar aquellos aspectos que se relacionan con los recursos empleados y la efectividad en los resultados para la salud de las personas y las poblaciones. Se presentan cinco posibles causas que pudieran explicar la multicausalidad del crecimiento de los costos en salud. Tres de ellas tienen la particularidad de que no aparecen suficientemente analizadas en estudios sobre el tema de los costos en salud y todas tienen un componente gerencial significativo: la corrupción, la deficiente organización de los servicios y el manejo irresponsable de los recursos por parte de los prestadores. Se amplía el análisis de la última causa mencionada al considerar que tiene mayor interés gerencial, ya que acumula un porcentaje alto de los costos y al mismo tiempo, es un elemento susceptible de modificarse por los gerentes en los niveles de atención primaria y secundaria. Para ampliar las posibilidades del análisis se procede a tratar la causa como problema y a este se le aplica el diagrama de causa-efecto de Ishikawa, que permite revelar las subcausas sobre las cuales los gerentes pudieran enfocar su trabajo para convertir la irresponsabilidad en corresponsabilidad. En la parte final se analizan cada una de las subcausas y se hacen recomendaciones sobre los métodos, habilidades y tecnologías gerenciales útiles para producir el cambio.

Palabras clave: Costos en salud, corresponsabilidad clínica, óptica gerencial, nivel primario de atención de salud, nivelo secundario de atención de salud. 


\begin{abstract}
This paper focused on health costs, particularly their increase, from a management approach. This implied to underline those aspects associated with the resources used and the effectiveness of results for the health of individuals and populations. Five possible causes that might explain the multi-causality of the health cost increases were presented. Three of them had not been deeply analyzed in the studies on health costs and had a basic management component: corruption, poor organization of service and irresponsible management of resources on the part of providers. The analysis of the last mentioned cause is extensive since it has a greater management interest, accounts for a high percentage of costs and it is at the same time an element that can be changed at the primary and secondary care levels by the managers. In order to extend the analytical possibilities, the cause was seen as a problem to which Ishikawa's cause/ effect diagram was applied. This allowed disclosing the subcauses upon which the managers might work to turn irresponsibility into co- responsibility. The last part of the paper analyzed each of these subcauses and made recommendations about the useful management methods, abilities and technologies to bring about the desired changes.
\end{abstract}

Key words: Health costs, clinical co-responsibility, management approach, primary health care, secondary health care.

\title{
INTRODUCCIÓN
}

Al tema de los costos en salud y particularmente a su crecimiento, se recurre frecuentemente en todos los estudios relacionados con la salud pública y sus limitantes. Para algunos, el lema "la salud no tiene precio" mantiene mucho interés político, sobre todo por su papel en las contiendas electorales cuando cada aspirante promete maravillas, pero en el fondo, la expresión "no tiene precio", se convierte en el camino más corto hacia la insostenibilidad de los sistemas de salud, cuando los razonamientos de tipo lógico y de base económica no se consideran. La influencia de la Economía de la Salud es muy importante en este debate, pues se orienta a evaluar los efectos de las intervenciones y no la intervención propiamente dicha, pero tampoco es la solución universal de este problema.

Lo real y aceptado es que la solución de los problemas de salud no depende solo de aportes monetarios y de infraestructuras, como ocurre en la producción industrial o en el comercio. Es algo mucho más complejo, en primer lugar, por reconocerse como un bien público y un derecho universal, en segundo, dado que implica tanto el derecho de las personas y las poblaciones, como su obligación para cumplir deberes como el autocuidado y los hábitos de vida saludables y en tercer lugar, y no menos importante, la producción de salud tiene un carácter social y en 
consecuencia desborda la capacidad y los recursos de los propios sistemas de salud. No se intenta tampoco convertir o querer convertir la salud en un negocio, pero nada impide que muchas de las excelentes tecnologías y habilidades que emplean los gerentes para hacer competitivos y eficientes sus negocios, puedan adaptarse para crear mejores sistemas y servicios de salud.

Cuando, por ejemplo, se examinan los "top"*de hospitales, aparece como un elemento fundamental el empleo de tecnologías gerenciales como explicación de haber alcanzado tal distinción. Una nota aparecida en la prensa española sobre los top 20 de hospitales de ese país (la mayoría de los galardonados son instituciones públicas), explica que:

Según los promotores del programa, los hospitales públicos incluidos en el TOP 20 tratan pacientes con una complejidad un 1,3\% mayor $y$, aún así, muestran unos índices de mortalidad y de complicaciones un 19 y un $24 \%$ menor que el resto de centros de su entorno. Por otra parte, el porcentaje de sustitución por cirugía mayor ambulatoria (CMA) es de un $57,3 \%$ en los hospitales TOP, frente al 49,9\% del resto de hospitales, lo que supone una diferencia positiva de 7,5 puntos porcentuales. Por otro lado, los centros hospitalarios reconocidos con este galardón han avanzado en la hospitalización de día y a domicilio, que en relación con la convencional resulta un $75 \%$ superior en los TOP en comparación con los demás centros. En general, los TOP 20 del Servicio Nacional de Salud muestran unos resultados mejores en la gestión de los recursos económicos, pues además de utilizar de forma más eficiente las camas hospitalarias, los centros galardonados han logrado unos costes de producción un $13 \%$ menor que los demás hospitales.

Los hospitales TOP demuestran que más gasto no se traduce necesariamente en mejor calidad. (El subrayado es del autor).

Según declaraciones del Conseller de Sanidad, Vicente Rambla en el gabinete de radio de la presidencia, la comunidad valenciana ha sido la segunda en número de hospitales participantes en el programa top 20. (Disponible en: http://www.san.gva.es/cas/inst/prensa/docs/top2003.pdf

Nótese, que al determinar los mejores hospitales, se consideraron no solo elementos clínicos y quirúrgicos, sino también otros relacionados con la optimización de los procesos de asistencia, el uso óptimo de los recursos disponibles, la aplicación de estrategias novedosas en cuanto al manejo de la hospitalización y la reducción de los costos de operación. Todo esto deja atrás los tiempos en que bastaba con la "satisfacción de los usuarios", con unas buenas estadísticas sobre el estado de salud de las personas, o el viejo concepto, todavía defendido por algunos, de que "curar implica buena calidad."

\section{LA MULTICAUSALIDAD EN EL CRECIMIENTO DE LOS COSTOS EN SALUD}

El crecimiento de los costos en salud, tiene un origen multicausal, como todo problema de salud, que se manifiesta de diferentes maneras y en diferentes combinaciones, según el país, el momento, la situación epidemiológica, las políticas sanitarias o el desarrollo económico.

Reducir el problema del crecimiento de los costos en salud a dos o tres indicadores o causas, es una forma errónea de simplificarlo, y además un riesgo cuando tales consideraciones están destinadas a fijar políticas sanitarias o a tomar decisiones de alguna envergadura. 
Por otra parte, el simple hecho de que los gastos sanitarios en relación con el PIB sean altos, o que su importe absoluto con respecto a otros países, también lo sea, no implica necesariamente que se hace mejor y más salud. Factores como el estado de salud de la población objeto, la presencia de factores de riesgo, la desigualdad en la distribución de los recursos, la forma en que estos se administran o lo acertado de las políticas sanitarias, solo para citar algunos, influirán para que estas "grandes"cifras tengan o no significado. "La combinación tóxica de malas políticas y mala economía es, en gran medida, responsable del hecho de que la mayoría de la gente en el mundo no pueda gozar de la buena salud que le es biológicamente posible."

Se analizan a continuación algunas de las posibles causas que influyen de manera significativa en los costos en salud, sin pretender abarcar todo lo que este tema incluye, pero con la intención de contribuir en algo a esclarecer este problema, no fácil de explicar y mucho menos de resolver.

\section{El complejo formado por mejor atención, mejores medicamentos y nuevas tecnologías}

Este es el aspecto "blando" en cuanto a crecimiento de los costos en salud. Si bien las metas del milenio aún no se alcanzan, es indiscutible que en muchos países y regiones se observan mejoras en la situación de la salud. La mayor supervivencia y mejor calidad de vida de los enfermos de cáncer, la reducción de enfermedades evitables por vacuna, la extensión con mayor o menor éxito de la atención primaria de salud, el mejoramiento de las tasas de mortalidad infantil, la reducción de muertes por causas evitables, los nuevos enfoques hacia la medicina familiar y la atención integral, pudieran justificar o al menos explicar en algunos casos, el crecimiento de los costos en salud.

En cuanto al futuro, algunas previsiones de crecimientos en costos de salud, se vinculan con una mayor o mejor atención a la salud. Según la Economist Intelligence Unit (EIU):

Unos cuantos de los principales mercados emergentes encabezarán el crecimiento de la inversión en servicios de salud y productos farmacéuticos. Brasil, China, India, México, Rusia y Turquía experimentarán un crecimiento del PIB real

considerablemente más rápido que el promedio global, de alrededor de $3 \%$ anual. Esto precipitará una seguridad social más amplia e integral, y mayores beneficios para más segmentos de la población. El sólido crecimiento económico generará ingresos más altos y permitirá mayor inversión doméstica en seguros médicos adicionales. $^{2}$

En cuanto a los medicamentos, mucho se discute actualmente sobre su influencia real o aparente en el crecimiento del gasto en salud. Algunos estudios demuestran que la mayor parte del aumento del costo farmacéutico, se debe realmente al incremento del número de recetas emitidas muy por encima del crecimiento de su precio medio. ${ }^{3}$ Esto puede asociarse con alguno o varios de estos factores: un volumen mayor de personas atendidas, la regulación de los precios por parte de los gobiernos y el empleo cada vez más amplio de genéricos en los servicios públicos de salud, esto último limitado por la política agresiva de los grandes consorcios farmacéuticos para demorar o impedir el lanzamiento de sus homólogos genéricos. ${ }^{4}$

El otro aspecto relacionado con los medicamentos se refiere a su efecto sobre la salud, por lo que se plantea que los nuevos productos y sus combinaciones, no producen en términos sociales, una elevación de los costos en salud, dado que 
reducen otros gastos sanitarios de mayor peso, tales como hospitalizaciones, intervenciones quirúrgicas o visitas reiteradas al médico.

Un ejemplo considerado ya clásico, se relaciona con la úlcera péptica:

A principios de los $90 \mathrm{~s}$, el costo anual de tratamiento con medicamento por persona era cerca de $\$ 900$ dólares, comparado con cerca de $\$ 28,000$ de la cirugía. El descubrimiento de que el $H$. Pylori es la principal causa de úlceras, llevó al uso combinado de antibióticos con los antagonistas $\mathrm{H} 2$ para su tratamiento, cuyo costo aproximado es de $\$ 140$ dólares por paciente. Estudios recientes, permiten establecer que el nuevo tratamiento ahorra por lo menos \$224 millones de dólares al año en costos relativos a las úlceras pépticas. ${ }^{5}$

Sin embargo, mejores medicamentos no implican automáticamente mejor salud ni menores costos. Por ejemplo, el abordar los problemas de salud a partir de los síntomas y no de sus determinantes y riesgos, implica mayor consumo de medicamentos y por tanto incremento de los costos.

El enfoque fármaco epidemiológico puede hacer una contribución notable a mejorar la relación costo efectividad en términos del empleo de medicamentos. Al hacer accesible a los prestadores toda la información relativa a sus efectos, composición, reacciones adversas, intolerancias, interacciones, uso racional, opciones, dosificación, precios y otros, facilitan decisiones terapéuticas más acertadas.

La situación de la tecnología es parecida a los medicamentos. La introducción de los avances en este campo solo se reconoce cuando tiene un impacto significativo en el diagnóstico, la prevención, la cura o la rehabilitación de los pacientes. El notable crecimiento de los gastos asociados al empleo del diagnóstico por imágenes, por ejemplo, puede asociarse perfectamente a impactos como los señalados anteriormente. Un estudio realizado en Argentina, muestra que en el período 2000/2004 la tasa de uso del diagnóstico por imágenes se elevó en el 46,8 \% mientras que las consultas crecieron solo en el $26,8 \%{ }^{6}$

Según un artículo publicado por The New England Journal of Medicine:

El advenimiento de la tomografía computarizada (TC) ha revolucionado el diagnóstico radiológico. Desde la introducción de la TC en los años 70, su uso se ha incrementado radicalmente. Se estima que más de 62 millones de escáneres con TC se realizan cada año en los Estados Unidos, incluyendo al menos 4 millones de niños. ${ }^{7}$

La introducción de las historias clínicas automatizadas en ambiente en red, la receta electrónica y la tarjeta individual magnética, han transformado significativamente el modo como se organizan y operan los servicios de salud, han logrado apoyar tecnológicamente la solución de problemas clásicos tales como la continuidad y la integración a escala de unidad y de región, han reducido desplazamientos de los prestadores y de los usuarios y sobre todo, han generado formas más eficientes de hacer salud y de conocer qué se hace y qué se puede hacer. Su impacto se ha detectado en diferentes partes del mundo desde hace más de quince años..$^{8-12}$

El complejo formado por mejor atención, mejores medicamentos y nuevas tecnologías, muestra características de proceso inversionista más que de gasto. El problema, una vez más, se relaciona con la habilidad de los lideres para hacer un uso racional, efectivo y eficiente de estas posibilidades, pues el éxito no es un 
producto natural ni espontáneo de la tecnología, son las personas hábilmente conducidas las que producen el efecto deseado.

\section{Transición demográfica}

La mayor esperanza de vida de las personas es producto de una combinación efectiva de los determinantes de la salud, en particular las condiciones y estilos de vida, el desarrollo económico y los avances en los sistemas y servicios de salud. El impacto cultural de esta misma combinación, ha tenido como efecto un descenso gradual de la natalidad. Estos factores se han traducido en un peso mayor de las personas con edades superiores a los 65 años, en el total de las poblaciones. Todo parece indicar que este proceso se mantendrá en la misma proyección, aunque posiblemente a un ritmo menor que el actual. Esta situación coloca, en primer plano, nuevos problemas que deben resolver los sistemas de salud, que incluyen la capacidad de respuesta para la atención a una población envejecida, con sus particularidades en términos de tratamiento y rehabilitación.

Según estudios encargados por la Unión Europea, el gasto promedio en salud que recibe una persona de más de 65 años cuesta de 2,7 a 4,8 veces el gasto promedio de una persona de 0-64 años. ${ }^{13}$ Esto significa que entre el 35 al $50 \%$ del gasto en salud en la vida de una persona se registra en esta última etapa, cuando generalmente deja de ser un ente productivo. En los próximos 20 años, los cambios en el tamaño y estructura de la población mundial incrementarán los costos de salud necesarios en el $14 \%$ en Europa y Asia Central, $37 \%$ Asia del Este y Pacífico, $45 \%$ Asia del Sur, $47 \%$ América Latina y el Caribe, $52 \%$ en África del Sur y $62 \%$ en Oriente Medio y África del Norte. ${ }^{13}$

Por su parte, la EIU, señala:

...la expansión y envejecimiento de las poblaciones impulsará la demanda de toda clase de servicios de salud durante el periodo 2008-12, y pondrá a prueba la capacidad de los gobiernos para proporcionarlos. En el mundo desarrollado, el costo de tratamientos de enfermedades relacionadas con la obesidad y los elevados índices de cáncer, combinados con el alto costo de las terapias a base de medicamentos controlados, plantearán un reto a las entidades que pagan por ellos, las cuales buscarán implantar controles de precios cada vez más estrictos y restricciones en el rembolso por medicamentos. El volumen, no el precio, hará que las ventas farmacéuticas se eleven durante el periodo que se pronostica (...) En el mundo en desarrollo, la incidencia de enfermedades crónicas, como hipertensión y diabetes, provocará una demanda altísima de tratamientos farmacéuticos. El gasto del sector salud se elevará en forma dramática en los principales mercados emergentes, al ritmo del crecimiento del PIB y de una mayor demanda. ${ }^{2}$

Según la experiencia holandesa, el $10 \%$ del total de gastos en salud estuvo asociado con el uso de la asistencia médica por personas en su último año de vida y el incremento de la longevidad, resultará en costos más altos debido a que las personas vivirán más tiempo. ${ }^{14}$

Para algunos especialistas, esta aseveración resulta en cierto modo tremendista, dado que tiende a explicar el crecimiento de los gastos de salud desde un solo enfoque y además, no considera, el acomodo o ajuste que los servicios deberán hacer para asimilar el cambio sin afectar la efectividad y la eficiencia.

Según un estudio realizado por el Centre de Recerça en Economia i Salut (CRES) de Barcelona: 
...es fácil darse cuenta de que las variaciones en el estado de salud de las personas mayores, no son el único factor que condicionará la relación futura entre envejecimiento demográfico y gasto sanitario (...) a este respecto, como veremos más adelante, puede resultar fundamental el grado en que se desarrollen nuevos dispositivos capaces de proporcionar una atención integral a las necesidades asistenciales de los ancianos, expandiendo y combinando óptimamente los actuales recursos sanitarios y sociales especializados (larga estancia, centros de día, atención domiciliaria, etc. . ${ }^{15}$

Lo cierto es que el escenario sanitario, social y económico cambió. Se trata entonces de que los sistemas y servicios de salud se reorganicen en función de ese nuevo escenario, para que encuentren soluciones sanitarias y gerenciales que se correspondan con las nuevas condiciones y demandas.

\section{Corrupción}

Cada año, el mundo gasta más de US $\$ 3.000$ billones en servicios de salud, la mayoría de los cuales son financiados por los contribuyentes fiscales. Este inmenso flujo de fondos resulta un objetivo atractivo para el abuso. Los riesgos son altos y los recursos preciosos: el dinero perdido por la corrupción podría ser utilizado para comprar medicamentos, equipar hospitales o bien contratar personal médico muy necesario. ${ }^{16}$

El informe global sobre la corrupción año 2006, estuvo centrado precisamente en el tema de la salud, y su contenido abarcó desde países altamente desarrollados como los Estados Unidos de Norteamérica o el Reino Unido hasta otros notablemente empobrecidos como Nigeria o Kenya, o tan ajenos a nuestra cultura como China. ${ }^{17}$

Entre las más notables manifestaciones de corrupción en el sector se mencionan: sobornos, manipulación de la información, hurto y malversación de recursos, sobreprecios de las empresas proveedoras de medicamentos y servicios**, manejo de los mecanismos de pagos, adulteración de medicamentos, desvío de fondos hacia proyectos "favoritos", falsas licitaciones y otros. Este escenario incluye tanto prestadores públicos como privados, proveedores y aseguradoras.

Hay otro tipo de corrupción que se pudiera llamar "implícita"y es la que tiene ver con los visitadores médicos. Fugh-Berman, un estudioso de este tema, dice que:

Un médico que pasa sólo un minuto con un representante de un laboratorio, termina, generalmente, prescribiendo un $16 \%$ más de los productos de dicho representante de lo que él o ella recetaba anteriormente. Pero, en un encuentro de cuatro minutos, la probabilidad de que recete más productos de esa firma se eleva a un $52 \% .{ }^{18}$ (La traducción es del autor).

Fugh-Berman imparte cursos en las escuelas de medicina y enfermería para entrenar a los futuros profesionales en como enfrentarse a esos vendedores, como parte de un programa que se viene generalizando a todas las escuelas de medicina de Estados Unidos.

Algunas de las medidas "organizativas"generan nuevas formas de corrupción, tal es el caso de los sistemas de retribución salarial. Por ejemplo, el pago por "servicios prestados", puede generar la indicación de estudios complementarios innecesarios o falsos, mientras que el pago por "paciente atendido"estimula la no provisión de servicios que este requiere. El salario fijo, sobre todo cuando es insuficiente, promueve la vocación hacia el soborno. Otro caso interesante es el de la descentralización, una idea excelente, pero amenazada por situaciones donde el 
dinero "baja" desde el nivel nacional, pero "se pierde" en algún punto intermedio del viaje, para aparecer milagrosamente en lujosas viviendas, enormes yates de recreo o cuentas en paraísos fiscales.

La unidad antifraude del Reino Unido, notificó que desde el año 1999 hasta la fecha ha logrado, como efecto de la reducción de la corrupción, recuperar para el sistema de salud, el equivalente a lo que se requeriría para la construcción de diez nuevos hospitales ${ }^{16}$ y considera que todavía falta bastante por recuperar.

Muchas de estas manifestaciones son consecuencia de la falta de transparencia de los sistemas de salud con respecto a los pacientes, sus familiares y la comunidad; la propia complejidad de los sistemas de salud, métodos de vigilancia en salud que no toman en cuenta aspectos económicos, materiales y financieros y el hecho de que la salud es una necesidad imprescindible para todas las personas.

La corrupción es una especie de fantasma que se oculta detrás de supuestos gastos en salud. No solo evita que los recursos lleguen a quienes deben ser beneficiados, sino además contribuye a la aparición de estadísticas engañosas en términos de gastos por PIB, bien ajenos a la realidad.

\section{Deficiente organización de los servicios, y errores en la toma de decisiones}

Una de las particularidades significativas de los servicios y sistemas de salud, es su capacidad para cambiar el escenario sobre el cual actúa, lo que debiera obligar a procesos consecuentes de reorganización que eviten que tales servicios se vuelvan inoperantes o simplemente ineficientes.

A la incompatibilidad entre el escenario y la organización, se unen la incapacidad para convertir buenos modelos en realizaciones prácticas, el empleo de métodos no participativos en el diseño de los servicios y la falta de un enfoque integral e integrador en su concepción.

Otro problema tiene que ver con las políticas sanitarias y las decisiones sobre su puesta en práctica. Muchos políticos prefieren las inversiones que promueven grandes titulares de periódicos, por ejemplo, el hospital con la última tecnología de punta o un centro para cirugía de transplante, en detrimento de acciones de promoción y prevención cuyos impactos no se notarían dentro de su periodo como gobernante, pero que implican y afectan a segmentos de la población significativamente mayores. Otras de las preferencias son las "reestructuraciones", que con frecuencia se orientan simplemente a cambiar lo que hizo el ejecutivo anterior, sea bueno o no, sin mayor criterio de efectividad o eficiencia y con los resultados negativos sobre el servicio y la comprensión de las personas y las poblaciones acerca de sus expectativas de atención.

Esto genera numerosos problemas, sobre todo incremento en los costos, debido a una baja relación costo efectividad a lo que se añade un sensible deterioro de la calidad. Estas son algunas de sus consecuencias: discontinuidad en la atención al paciente, tiempos y desplazamientos excesivos o innecesarios, falta de sincronización entre servicios, alto nivel de redundancia informativa o administrativa, no correspondencia entre la forma en que se ejecutan los procesos y el contenido de las políticas y estrategias del sistema; exceso de niveles intermedios entre el hecho sanitario, social o económico y la alta dirección; bajo nivel de eficiencia y de efectividad, variabilidad de la práctica clínica, barreras a la accesibilidad, inoportunidad y baja capacidad de respuesta en la generación de los servicios, uso inadecuado de medios diagnósticos y de medicamentos, entre otros. 
La solución de la mayoría de estos problemas compete a las esferas intermedias y superiores de dirección de los sistemas de salud, niveles donde se crean y se implementan los modelos y las reformas. Otros pueden resolverse mediante acciones de las direcciones de los hospitales y de los centros de atención primaria, pero siempre dentro del rango y las limitaciones que les impongan reformas o modelos no estimulantes para la creatividad.

\section{Manejo irresponsable de los recursos por parte de los prestadores}

Según Cosialls Delhi, ${ }^{19}$ el $70 \%$ del costo hospitalario está representado por las decisiones clínicas (...) (cultivos de Gram, espirometría, placas y otros), lo cual expresa claramente el peso y significación económica de tales decisiones. En los servicios de atención primaria, posiblemente esta proporción sea mayor. Si no se considera la relación costo efectividad de estas decisiones, su implicación negativa sobre los gastos en salud, resulta significativa.

En ese sentido Moreno Rodríguez señala:

...por cualquier pretexto, se indican hoy en el mundo análisis, radiografías, endoscopias, ultrasonidos, gammagrafías, tomografías y resonancias -por no citar más que algunos-, que lo único que hacen es multiplicar los costos y aumentar los errores. ${ }^{20}$

En ese mismo artículo, Moreno Rodríguez, muestra que un estudio realizado en su propio hospital y no concluido aún, se detectó que "de 945 análisis de todo tipo realizados a 49 pacientes (un promedio de 17 análisis por enfermo hospitalizado), aproximadamente el $25 \%$ se consideraron innecesarios."

En entrevista realizada a Pastor Castell-Florit, *** sobre estos temas, comenta que durante su trabajo como Director de Salud de la Provincia Habana y posteriormente de la capital del país, realizó estudios sobre los resultados de radiografías y análisis clínicos en diversas instituciones hospitalarias y de atención primaria del territorio a su cargo. En el caso de los primeros, los resultados negativos alcanzaban como promedio el $45 \%$ y en los segundos un poco menos del $48 \%$. Un mejor trabajo en el empleo del método clínico y en la toma de conciencia de médicos y directivos, permitió reducir estas cifras a valores entre el 10 y el $15 \%$, con un sensible impacto en el consumo de recursos y en los costos.

En Argentina, una investigación en entidades privadas, que incluye a 870000 beneficiarios con una media de edad de 33,6 años, de los cuales el 14,9\% es mayor de 60 años, encuentra que cerca del $80 \%$ de los estudios hechos en el servicio de resonancia magnética, no muestran resultados anómalos. ${ }^{6}$

El impacto de los medios diagnósticos basados en imágenes, representa uno de los avances más significativos de la medicina en los últimos años. Sin embargo, su uso indiscriminado, ya sea por practicar una medicina defensiva, por estar a la moda, complacer al paciente o simplemente convertirla en la primera opción diagnóstica, implica crecimiento de los costos y sobre todo arriesgar la salud de las personas.

Los estudios epidemiológicos han aportado evidencias directas a favor de que las dosis recibidas por los órganos explorados mediante una serie de TAC (dos o tres exposiciones y las correspondientes placas) incrementan el riesgo de desarrollar un cáncer. La evidencia es razonablemente convincente para los adultos y muy convincente para los niños. ${ }^{7}$ (La traducción es del autor).

Por otra parte, y más grave aún: 
...si es cierto que alrededor de una tercera parte de todas las tomografías diagnósticas realizadas no están justificadas, y todo indica que es así, posiblemente 20 millones de adultos y lamentablemente, más de un millón de niños por año, en los Estados Unidos están siendo irradiados innecesariamente. (La traducción es del autor).

Se trata entonces de entender lo que representa tanto en términos de costos, como de efectividad clínica, las facultades otorgadas al médico para crear e inducir demanda y por tanto influir en cuáles, dónde y cómo, se emplean los recursos sanitarios. Los problemas más críticos asociados a estas facultades, se relacionan con los medios diagnósticos y con la prescripción farmacéutica. En el primer caso, tanto el sobreempleo como el subempleo generan efectos negativos en la calidad del servicio, en la salud del paciente y en los costos sanitarios. En el segundo caso, la complacencia, el desconocimiento, el síndrome de la "última generación", la falta de acceso a las mejoras opciones o las fallas en la ética, agravan sensiblemente tanto los costos como los problemas de salud. ${ }^{21}$

Lo importante es comprender que el dilema de los costos de la salud no es solo un problema de efectividad, es también un problema de eficiencia, y en la medida que se valoren ambos aspectos al nivel del prestador directo, la relación entre dinero empleado y resultados en salud será muy superior. A continuación se profundizará en este aspecto.

\section{NECESIDAD DE PASAR DE LA IRRESPONSABILIDAD A LA CORRESPONSABILIDAD}

\section{La debida correspondencia entre autoridad y responsabilidad}

La autoridad y la responsabilidad son conceptos parecidos pero con implicaciones opuestas. Mientras que la primera se refiere a la capacidad formal, adquirida o natural para hacer o mandar a hacer, la segunda tiene que ver con la obligación que tiene el portador de la autoridad para responder ante quien se la otorgó, o ante la sociedad, por el uso que ha hecho de ella y los resultados que ha obtenido.

Es de suponer que debe existir un equilibrio entre la responsabilidad que se exige y la autoridad que se ha otorgado, lo que significa que nadie está obligado a responder más allá que lo que le ha sido autorizado a hacer o mandar a hacer. En gerencia, esta relación se considera un principio que se enuncia de la forma siguiente: Correspondencia entre autoridad, medios y responsabilidad. Como dato curioso, este es uno de los pocos principios de esta disciplina que no ha desaparecido ni tampoco ha sido modificado por los profundos cambios de los últimos años. A continuación se expresa como se manifiesta este principio en el trabajo clínico.

- En las organizaciones de servicios, el personal de contacto tiene la máxima responsabilidad en la operación, dado que conjuntamente con el cliente, y en el momento en que se encuentran (nunca antes ni después), se produce y materializa el hecho transaccional. La calidad depende de quien proporciona el servicio, cuándo, dónde y cómo lo hace, es inseparable de su prestador. Por ejemplo, el masajista en un centro de belleza, la aeromoza en un avión en vuelo o el carpetero en un hotel, materializan el hecho económico o social durante el proceso de contacto, aunque no van más allá de "sugerir" otros productos o servicios. 
- Los hospitales y los centros de atención primaria, son también organizaciones de servicios, pero se caracterizan por que el personal de contacto, particularmente médicos y enfermeras, no solo opera el servicio directamente con el cliente -en este caso paciente- sino además tiene las facultades siguientes:

- Crear el servicio.

- Inducir la demanda.

- Decidir entre opciones.

- Movilizar recursos.

- Crear trabajo para otros servicios.

- Por tanto, el personal de contacto de las organizaciones de salud, no solo realiza procederes clínicos, sino también decide sobre recursos, sin que esté necesariamente obligado a utilizarlos con la misma efectividad o eficiencia que le corresponde a un gerente. Lo más complejo de la situación descrita, es que nos referimos a una proporción que alcanza alrededor del $80 \%$ del costo total de los recursos gastables que se emplean en la atención a la salud.

- Es curioso, además, que la provisión de medicamentos, la imaginología o el laboratorio clínico, por solo citar estos casos, dependen en la práctica, de una demanda que crea el prestador directo, la cual está en función de su nivel de actualización profesional y técnica, de cómo se ajuste a las normas y prácticas generalmente aceptadas, del nivel de equidad y ética con que realice su trabajo y de su estilo personal de actuación.

Es indiscutible que todas estas atribuciones deben mantenerse, pues son inherentes al trabajo del médico. De hecho, es la práctica médica la que determina los resultados y los recursos que serán utilizados para lograrlos, eso no hay que cambiarlo. Por otra parte, el principio de la correspondencia entre autoridad, medios y responsabilidad no se cumple, pues quien más influye y determina en el consumo de recursos sanitarios, no está obligado a responder por su uso racional.

No se trata, por tanto, de restar autoridad, si no de ampliar la responsabilidad asociada, lo que implicaría que el clínico, en sus decisiones, tuviera en cuenta el uso más adecuado de los recursos tecnológicos, humanos, materiales y organizativos, en función del mejor y menos efecto riesgoso sobre la salud de las personas que atiende. Este enfoque es una parte integrante de la llamada "gestión clínica" que es una versión españolizada del clinical management del idioma inglés. El término resulta un poco confuso, por lo que es preferible hablar de corresponsabilidad clínica, para hacer la denominación más consecuente con el principio gerencial que la sustenta.

En definitiva, lo más importante no es si los costos de la salud se elevan o descienden, lo esencial consiste en lograr que los que se utilicen rindan el máximo en términos de mejorar la salud, la calidad de vida y el bienestar de las personas, y para lograrlo, habría que incorporar el componente gerencial al trabajo del clínico. Se trata entonces, de combinar el concepto de efectividad, que se mide en términos de la práctica médica, con el de eficiencia, que se mide en términos de cuanto se ha hecho con los recursos de que se dispone. De esta forma, tendría sentido hablar de inversión en salud en vez de gastos en salud. 
Si se acepta que el manejo irresponsable de los recursos por los prestadores es una de las causas de mayor peso relacionadas con los costos en salud, habría que analizar como es posible revertir la situación, pero sin convertirlo en órdenes, resoluciones o mecanismos de persecución para los prestadores.

\section{La irresponsabilidad clínica como problema gerencial}

Una manera gerencial de abordar esta causa, pudiera ser tratarla como problema. De esta forma, resultaría posible emplear, para su solución, herramientas que han sido validadas en la práctica de las organizaciones de éxito. Esta causa, expresada en forma de problema, la denominaremos "bajo nivel de corresponsabilidad en la práctica clínica", de manera de disponer de una expresión menos absoluta y más fácil de aceptar.

Las técnicas de análisis y solución de problemas siguen, generalmente, un esquema como el siguiente:

1. Determinar si el problema efectivamente lo es, si es suficientemente importante como para trabajar en su solución y si tiene posibilidades de ser resuelto. Estos tres aspectos se cumplen en este caso.

2. Descomponer el problema sucesivamente en los factores que inciden en su aparición, hasta llegar a los más elementales.

3. Jerarquizar los factores encontrados, dado que generalmente, un pequeño grupo de ellos son suficientes para determinar el problema en su casi totalidad (Principio de Pareto que indica que el $20 \%$ de las causas producirá el $80 \%$ de los efectos, mientras que el $80 \%$ restante solo cuenta para el $20 \%$ de los efectos). Este es un proceso parecido a cuando se establecen las cinco o diez primeras causas de muerte al estudiar la mortalidad.

4. Definir las estrategias y las acciones que se seguirán para eliminar el problema, para reducirlo o para que su efecto negativo sea menor.

Para la ejecución del segundo paso, se propone emplear el diagrama de causaefecto de Ishikawa que permite crear una imagen visual bastante fácil de comprender de cuantos y cuales factores (en este caso, causas y causas de causas) inciden en este problema, pero sin expresar aún el peso específico que cada una tiene en su manifestación. El que aquí se presenta, tiene solo un carácter genérico $\mathrm{y}$, por tanto, es útil únicamente en términos de apreciar la herramienta, pero cada institución o servicio de salud confeccionará el propio, que debe incluir la jerarquización de acuerdo con su propio escenario (figura). 


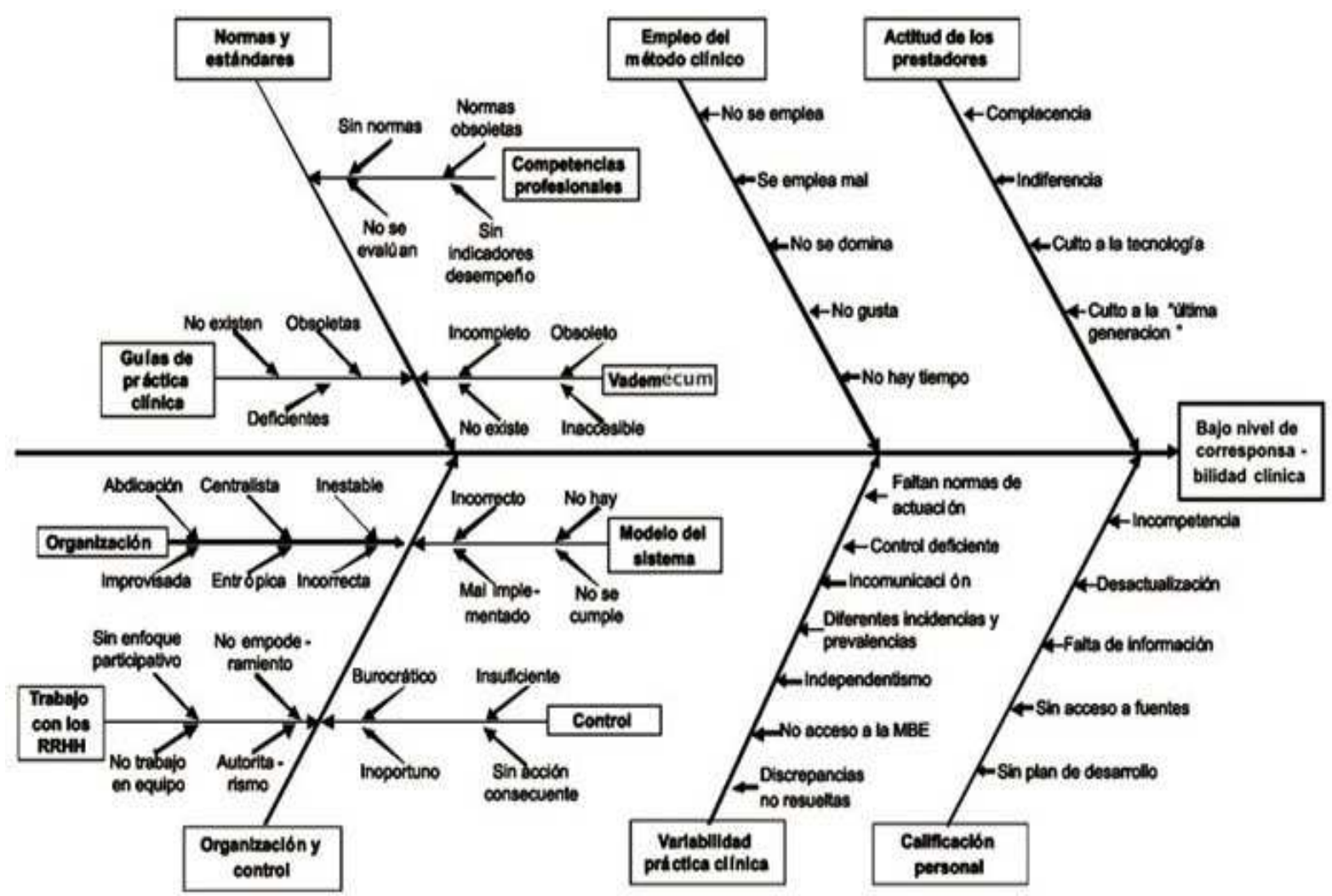

Nota: El diagrama causa-efecto es más conocido como "espina de pescado" por la forma que adopta su figura. Esta herramienta fue aplicada por primera vez en 1953 por Kaoru Ishikawa, profesor de la Universidad de Tokio, con vistas a integrar los diferentes criterios que tenian los ingenieros de una fábrica con respecto a los problemas de calidad. Su uso se ha generalizado en todas aquellas situaciones en las que se requiere apreciar, con claridad, las relaciones entre un asunto o un problema y las posibles causas que contribuyen a que se manifieste.

Fig. Diagrama causa-efecto sobre "bajo nivel de corresponsabilidad clínica."

\section{CARACTERIZACIÓN DE LOS FACTORES Y POSIBLES ACCIONES}

En la imposibilidad de resolver en el marco de este trabajo y en términos globales, los pasos 3ro. y 4to. previstos en las técnicas, se presenta a continuación una serie de elementos que permiten comprender mejor las causas que se muestran y las habilidades y tecnologías gerenciales que se podrían aplicar en cada caso y que debieran servir de guía para el trabajo práctico. Están organizados siguiendo los seis factores que aparecen en el primer nivel de descomposición, mostrado en la figura.

\section{Actitud de los prestadores}

Se refiere a como las personas enfrentan las situaciones en la práctica. La experiencia personal, las costumbres, la ideología, la ética o la moda, generan comportamientos que con frecuencia desbordan las mejores intenciones de sistemas o de modelos. La complacencia es un buen ejemplo, hace que el prestador "caiga bien". La mamá prefiere para su niño un medicamento caro en vez de suero oral para las diarreas, el diabético desea repetir su TAC pues sospecha que tiene nuevos padecimientos que no aparecieron en el control de salud realizado el mes pasado, la simpática anciana, que no falta a una consulta, quiere una batería completa de análisis clínicos pues tal vez padezca anemia, además de varias 
recetas para completar su botiquín. El clínico complaciente resuelve y los costos se cargan al seguro o al servicio público de salud, según sea el caso, pero ambos son costos sociales. Los problemas de comportamiento no se resuelven con medidas de ordeno y mando. Un enfoque gerencial participativo, donde los prestadores directos se familiaricen con el problema y se incorporen a los procesos de toma de decisión, puede ayudar a crear actitudes positivas en cuanto a la mejor forma de usar sus atribuciones para establecer el diagnóstico e indicar el tratamiento. Este enfoque, debe completarse con la creación de estándares o guías en cuya confección participarían esos prestadores y con técnicas de control selectivo y por excepción, para comprobar si se ha tenido éxito o no.

\section{Empleo del método clínico}

En el proceso del diagnóstico, la clínica -es decir, el interrogatorio y el examen físico precede y guía al laboratorio. Son dos procederes diferentes e independientes, pero íntimamente relacionados. La clínica es lo primero. Así como el interrogatorio guía al examen físico y, por tanto, un médico realmente profesional siempre interroga antes de examinar, así también el cuadro reduce las posibilidades diagnósticas y por eso guía y racionaliza el uso de la tecnología. Sencillamente, el proceso no puede ser a la inversa. ${ }^{20}$

El rango de causas del no empleo del método clínico es muy amplio, como se aprecia en la figura. Abarca desde fallas en la formación de los médicos, hasta carga asistencial demasiada alta que propicia la tendencia de desviar el problema hacia otros servicios o medios, para poder cumplir con el número de pacientes que debe atender en una jornada.

La alarma ya ha empezado a sonar, sobre todo en muchos casos, donde el método clínico bien empleado, reduce significativamente la necesidad del empleo de tecnologías agresivas, o en otros donde además del método clínico, pueden emplearse con igual efectividad, tecnologías menos riesgosas para la salud del paciente.

Hay una considerable literatura cuestionando el empleo de TAC, o el uso de múltiples escaneos con TAC, en una variedad de contextos, incluyendo el manejo de lesiones contusas, crisis epilépticas y dolores de cabeza crónicos y sobre todo, se cuestiona su empleo como herramienta para el diagnóstico primario de apendicitis en niños (..... ${ }^{7}$ (La traducción es del autor).

En un sondeo de opinión a radiólogos pediátricos, consideran que posiblemente una tercera parte de los estudios con TAC, puede ser sustituido por enfoques alternativos o simplemente, no ser ejecutados en lo absoluto, ${ }^{, 22}$ y esto depende del profesional que firma las órdenes de estudios complementarios.

No se trata de enfrentar la tecnología con el método clínico, lo que sucede es que el segundo, representa el ejercicio real y efectivo de la medicina y en consecuencia, asegura un uso racional de la tecnología disponible para los sistemas y servicios de salud, en el mundo actual.

Visto desde el punto de vista gerencial, la solución de las fallas en el empleo del método clínico requiere resolver las debilidades en los problemas de formación profesional, estar al tanto de los hechos que demuestran su poco empleo, una organización adecuada del servicio para que el médico disponga de un tiempo racional para desempeñarse bien y buenos mecanismos de motivación y comunicación con el personal. 


\section{Normas y estándares}

Todo proceso gerencial requiere el establecimiento previo de las metas, normas, estándares u objetivos que constituyen la guía y el marco dentro de los cuales se ejecutarán las operaciones y sean al mismo tiempo la referencia contra la cual deben compararse los resultados para determinar si se alcanzaron o no.

Los sistemas y servicios de salud, tienen entre sus características un margen muy amplio para el establecimiento de estándares, algunos incluso con rango internacional. Los diagnósticos, las enfermedades, los procedimientos clínicos y quirúrgicos, el empleo de medicamentos, sus interacciones y resultados adversos, son solo un ejemplo del amplio marco de esta posibilidad.

La ausencia de normas y estándares justifica en buena medida la diversidad de actuaciones ante un mismo problema, la mayor posibilidad de decisiones erróneas, menos efectivas o menos eficientes; la dificultad para identificar la mejor práctica para un caso concreto, la imposibilidad de evaluar el desempeño o la competencia del profesional actuante, entre otros.

Sin normas básicas, tales como las guías de práctica clínicas, las normas técnicas de competencias profesionales y catálogos completos de manejo de medicamentos (efectos clínicos, interacciones, intolerancias, presentaciones, efectos adversos, predisposiciones, mejores opciones de dosificación, precios y otras opciones), no hay forma de posibilitar y exigir que los prestadores se ajusten a la mejor práctica o al menos, a la que ha sido aceptada como la mejor en la institución correspondiente.

La existencia de normas y estándares es una condición necesaria para mejorar la calidad de las decisiones del médico actuante, pero para ello, se considerarán algunos requerimientos:

- Las normas y estándares son creadas en procesos interactivos con la participación de quienes tienen que aplicarlas.

- Están fácilmente a la disposición del prestador, para el momento en que requiere aplicarlas.

- Las diferentes formas que adoptan las evaluaciones: auditoría médica, control de calidad, comités hospitalarios, evaluación del desempeño, entre otras, utilizan las normas y estándares como sus referencias.

- Los resultados de las evaluaciones generan acciones consecuentes, ya sea el premio, la llamada a la atención o la modificación de la norma cuando la práctica real demuestra su inoperancia.

\section{Organización y control}

La organización y el control son la base fundamental de todo trabajo gerencial y dos de los elementos que más condicionan el éxito de una institución. Ambos crean el espacio físico y conceptual, dentro del cual se ejecutan todos los procedimientos, operaciones y flujos de trabajo. Su diseño es un trabajo técnico bastante complejo, y es recomendable que lo acometan especialistas.

La existencia o no de un modelo de atención a la salud, es el primer elemento a considerar. Un modelo refleja la concepción más general de cómo debe hacerse la 
organización y el control. Descansa en definiciones fundamentales, en políticas generales y en estrategias básicas, y aunque todo esto es necesario, no es suficiente. El diseño puede ser erróneo, inoportuno o incompleto y esa condición arrastra todo lo demás. Incluso, de no tener ninguno de estos defectos, puede fallar al momento de implementarse sobre todo si es de ucase, sin que los posibles ejecutores entiendan qué es, porqué es bueno y cuál es la contribución que de ellos se espera.

El control tiene una tendencia, casi universal, a burocratizarse y los sistemas y servicios de salud lo hacen con mucha facilidad. Si se espera la contribución de los clínicos, no tiene sentido atiborrarlos de procedimientos administrativos que les impiden hacer su real trabajo.

Los estilos de dirección representan otro problema. En el mundo actual, prevalece el enfoque participativo, el empoderamiento y los equipos autodirigidos. Si se quiere lograr que los clínicos asuman una responsabilidad mayor, fundamentalmente gerencial, por sus decisiones diagnósticas o terapéuticas, hay que elevar entonces su protagonismo en los procesos en que participa y en las decisiones de las cuales serán objeto y sujeto.

\section{Variabilidad de la práctica clínica}

La variabilidad de la práctica clínica es un tema muy investigado por sus implicaciones en la efectividad de la atención médica, en el empleo de recursos y en los costos en salud. Su mayor complicación reside tanto en el gran número de variaciones que se pueden encontrar, como en las razones que pretenden explicarlas.

Se ha descrito, por ejemplo:

...variaciones en las tasas de implantación de prótesis de cadera de hasta seis veces entre trece estados de los EUA. La diferencia aumentaba a 26 veces para el caso de las esclerosis hemorroidales (...) En 1984, se realizó un estudio que medía la probabilidad de que a un niño residente en Vermont (EUA), le fuera practicada una amigdalectomía durante su infancia y se observó que esta difería hasta en nueve veces, en dependencia del hospital en que le correspondiera la atención sanitaria, siempre dentro del mismo estado. ${ }^{23}$

El diagrama de la figura, intenta caracterizar un número de causas posibles, pero de ninguna forma las abarca todas. Algunas de ellas, son fáciles de comprender e incluso relativamente sencillas de resolver, tal es el caso de la carencia de normas y estándares y el deficiente control, los cuales ya se trataron en un inciso anterior.

La incomunicación, el independentismo y las discrepancias no resueltas, pueden originarse en la tendencia natural de los profesionales de salud al autodesarrollo, pero si este último no se incorpora socialmente dentro de la institución, se crean islas de conocimientos. La solución de estos problemas está en el trabajo en equipo, la introducción de sesiones y eventos científicos abiertos al debate amplio y la capacidad de los líderes para estimular la iniciativa y la creatividad.

Las diferencias en cuanto a la incidencia y la prevalencia de las enfermedades, pueden explicar y hacer aceptables algunas variaciones, pues responden a la capacidad del profesional de la salud para actuar en función del paciente y sus circunstancias. 
La medicina basada en la evidencia (MBE) ha sido definida como el uso consciente, explícito y juicioso de la mejor evidencia actual, para hacer decisiones acerca del cuidado de pacientes individuales. Hay dos elementos significativos en la definición, una se relaciona con presentar la mejor evidencia hasta ahora, con respecto a un proceder clínico, diagnóstico, terapéutico o quirúrgico, de manera de apoyar el trabajo del médico. El otro, se refiere a la decisión del médico, para el cual la evidencia es solo un componente que se añade a su talento y experiencia, y en función de ellos decide la conducta a seguir en un caso específico.

La MBE no sustituye, de ninguna manera el talento, la maestría y la experiencia del médico:

Sin maestría clínica, existe el riesgo de que la práctica llegue a estar tiranizada por la evidencia, pues incluso una excelente evidencia externa puede ser inaplicable o inapropiada para un paciente concreto. Sin la mejor evidencia actual, el riesgo es que la práctica quede rápidamente desfasada en perjuicio del paciente. ${ }^{24}$

Esta afirmación explica claramente la necesidad de un acceso fácil y sistemático a los trabajos de la MBE, que es el camino más directo para contribuir a la actualización del profesional, familiarizarlo con las mejores prácticas y en consecuencia reducir la variabilidad no justificada en la práctica clínica.

\section{Calificación personal}

Generalmente, los profesionales de la salud, generan su propio conocimiento, lo desarrollan en la práctica y lo personalizan, por tanto, su formación no representa un serio problema para las organizaciones sanitarias. El punto crítico está en cómo se socializa esa preparación en función del modelo, los objetivos y las estrategias de la organización, en lo que se conoce como gestión del conocimiento.

El trabajo en equipo, la creación de normas y estándares en ambiente participativo, la celebración de talleres de trabajo para encontrar soluciones a los problemas, el desarrollo de eventos científicos sin restricciones ni prohibiciones administrativas, el acceso a fuentes fundamentales para el profesional de la salud, tales como los sitios y las bases de datos disponibles en Internet, forman el fundamento ideal para crear un conocimiento corporativo, que es un valor de la organización como un todo. Ese conocimiento corporativo, al reflejar los intereses de la organización, contribuye a fortalecer la corresponsabilidad de los clínicos con respecto al manejo de los recursos.

Por supuesto que se requieren otras vías. Los planes de capacitación generalmente no son muy efectivos, pero pueden ayudar si están bien concebidos y en función de los intereses de la organización, sobre todo cuando se entrenan las personas en técnicas de análisis y solución de problemas, trabajo en equipo y enfoque participativo.

La incompetencia generalmente es individual, salvo cuando se van a hacer cambios fundamentales que abarcan la organización como un todo, por ejemplo, la introducción de historias clínicas informatizadas en ambiente en red, que demanda que los implicados sean habilitados con todas las competencias que requieren para su uso.

\section{UN COMENTARIO FINAL}

http://scielo.sld.cu 
No puede hablarse de inversión en salud mientras muchos de los gastos que los integran se originen en políticas y modelos erróneamente concebidos, deficiente organización de las instituciones y de los servicios, mal manejo de los fondos que fueron destinados a hacer más salud, decisiones acerca de medicamentos y tecnologías que no se justifican en términos epidemiológicos, clínicos o gerenciales, falta de una conciencia de los prestadores directos acerca del uso más racional de los recursos; y carencia de normas y estándares que apoyen la operación y sean los referentes para una evaluación acertada. No se trata de alcanzar altos porcentajes de costos en salud con respecto al PIB, lo que se requiere es convertir recursos en resultados, el cual es uno de los principios imbatibles de la gerencia contemporánea.

Si los gastos en salud, se manejaran con suficiente transparencia, efectividad y eficiencia, dejarían de ser un problema para convertirse en una inversión social. Esto repercutirá en menores gastos futuros y sobre todo en una población mental y físicamente mejor preparada y, por tanto, en condiciones superiores para ser más productiva, más creativa y más dispuesta para el disfrute pleno del milagro de la vida.

Los prestadores directos pueden hacer mucho en esta dirección. Si se toma en cuenta que sus órdenes diagnósticas y terapéuticas generan, justamente o no, el componente mayor de los costos en salud, es fácil deducir que un compromiso mayor de esos profesionales acerca de decisiones más racionales y acertadas con respecto a los recursos implicados en sus decisiones, permitiría hacer salud para más personas y hacer mejor salud para todos. El concepto de corresponsabilidad clínica se basa en esta percepción.

Ahora bien, la corresponsabilidad clínica actúa a nivel de la prestación directa del servicio, pero muchos otros costos evitables se originan en el resto de los niveles y procesos del sistema de salud, e incluso pueden hacer inútil cualquier intento de mejorar el desempeño del prestador, como es el caso de la centralización, o el de políticas y estrategias erróneas. La forma de hacerlo excede las posibilidades de este artículo, pero es conocido que la orientación hacia los determinantes sociales de la salud, el enfoque promocional y preventivo, el enfoque sistémico epidemiológico, la intersectorialidad y la concepción integral para el diseño y operación de modelos de salud, forman parte de las posibilidades que existen, son conocidas y pueden ser enseñadas y aprendidas.

\section{REFERENCIAS BIBLIOGRÁFICAS}

1. Marmot M. Lo que determina la buena salud [sitio en Internet]. 2008[citado 28 Ago 2008]. Disponible en:

http://news.bbc.co.uk/hi/spanish/science/newsid $7585000 / 7585818 . s t m$

2. Economist Intelligence Unit. Servicios de salud, a todo lo que dan. Periódico digital La Jornada. 24 de junio 2008 [sitio en Internet]. [citado 18 Jun 2009]. Disponible en:

http://www.jornada.unam.mx/2008/06/24/index.php?section=economist\&article $=0$ $\underline{24 n 1 e i u}$

3. El mercadeo del medicamento en España. Boletín de coyuntura [sitio en Internet]. Dic 2005 [citado 28 Ago 2008]: 8. Disponible en:

http://www.farmaindustria.es/Farma Public/index.htm 
4. The bitterest pill. The Economist. New York, 2008 Jan 24.

5. AMIIF. El acceso a la salud: más allá de los precios: una responsabilidad compartida [sitio en Internet]. [citado 2009]. Disponible en:

http://www.amiif.org/cms/images/stories/pub amiif/p accesosalud.pdf

6. ADEMP. Evolución de los costos en las entidades de cuidado de la salud, 2000/2004 [sitio en Internet]. [citado 2009]:1. Disponible en:

http://www.ademp.com.ar/estadistica/costos\%201.pdf

7. Brenner DJ, Hall EJ. Computed Tomography -An increasing source of radiation exposure. The New England J Med. 2007;357(22):2277-84.

8. Servicio Andaluz de Salud. Experiencia de integración en historias de salud. Proyecto Diraya. Andalucia: Servicio; 2002.

9. Águila A, Valenzuela P. Experiencias del registro electrónico de pacientes en un servicio universitario de neonatología. Rev Méd Chile. 2005;133:241-5.

10. Flory J. Health care turning to electronic files. EE.UU. Columbia Daily Tribune. 2006 Jan 8.

11. Sabaté J. Qué es la receta electrónica. Periódico digital CONSUMER.ES EROSKI, 16 de enero de 2007. Cataluña, España [sitio en Internet]. [citado 4 Sept 2008 ]. Disponible en: http://www.consumer.es/hemeroteca/portadas/2007/01/16/

12. Protti D. Comparison of information technology in general practice in 10 countries. Canadá. Healthcare Quaterly. 2007;10(2):107-16.

13. UE. European Economy. The impact of ageing on public expenditure 20042050. Special report No. 1/2006 [sitio en Internet]. [citado 28 Jul 2008]. Disponible en: http://ec.europa.eu/

14. Polder JJ, Barendregt JJ, van Oers $\mathrm{H}$. Health care costs in the last year of lifeThe Dutch experience. Social Sci \& Med. 2006;63(7):1720-31.

15. Casado D. Los efectos del envejecimiento demográfico sobre el gasto sanitario: mitos y realidades. Gac Sanit. 2000;15(2):154-163.

16. Transparency International. Informe global de la corrupción 2006 [sitio en Internet]. [citado 8 Sept 2008]. Disponible en:

http://www.eyt.org.ni/Informes/gcr2006 espanol.pdf

17. Burns RA. La corrupción en el sistema de salud chino. La Gran Época [sitio en Internet]. [citado 2008]. Disponible en: http://www.lagranepoca.com/articles/2007/08/23/1109.html

18. Weintraub A. Just Say No to Drug Reps. Business Week. 2008 Jan 24.

19. Cosialis D, Cosialis IP. Gestión clínica y gerencia de hospitales [editorial]. Madrid-Barcelona: Harcourt Brace de España, S. A.; 2000.

20. Moreno Ramírez MA. La clínica y el laboratorio. Rev Cubana Med. $2000 ; 39(4): 265-70$. 
21. Carnota O. Tecnologías gerenciales: una oportunidad para los sistemas de salud. Panamá: Universidad de Panamá; 2005.

22. Slovis TL, Berdon WE. Panel discussion. Pediatr Radiol. 2002;32:242-4.

23. Gumero J, Rubio S, Repullo J. Economía de la salud: instrumentos. Madrid: Díaz de Santos; 2006.

24. Sackett $D$. Medicina basada en la evidencia: lo qué es y lo qué no. BMJ.1996;312:71-2.

* "Top"es una palabra inglesa que se refiere a los más destacados en alguna esfera. En el campo de los sistemas de salud se emplean técnicas de referenciación competitiva (benchmarking), para determinar los hospitales o los centros de atención primaria que pueden considerarse los mejores en su campo. A continuación de la palabra, se acostumbra colocar el número que representa cuantos clasifican como los mejores. Por ejemplo, TOP 10 significa que solo se consideran los diez primeros.

** Los Estados Unidos de Norteamérica gastan el 15,3\% de su PIB en servicios de salud, pero se considera que entre el 5 al $15 \%$ de ese presupuesto se evapora en sobreprecios.

*** Entrevista hecha por el autor al Dr. C. Pastor Castell-Florit Serrate, Director de la Escuela Nacional de Salud Pública, para conocer sus experiencias como Director Provincial de Salud, en el manejo racional de los medios diagnósticos. La Habana, 28 de agosto de 2008.

Recibido: 28 de mayo de 2009.

Aprobado: 27 de noviembre de 2009.

Orlando Carnota Lauzán. Escuela Nacional de Salud Pública. Calle Línea esq. a I. El Vedado 10400. La Habana, Cuba.

E-mail: orlando@infomed.sld.cu 